\title{
Les esclaves dans les petites annonces des journaux brésiliens au dix-neuvième siècle : discours et idéologie
}

Slaves and Classified Ads in 19th Century Brazilian Newspapers: Discourse and Ideology

Helena Nagamine Brandão

\section{Q OpenEdition Journals}

Édition électronique

URL : http://journals.openedition.org/aad/1230

DOI : $10.4000 /$ aad. 1230

ISSN : 1565-8961

Éditeur

Université de Tel-Aviv

Référence électronique

Helena Nagamine Brandão, « Les esclaves dans les petites annonces des journaux brésiliens au dixneuvième siècle : discours et idéologie ", Argumentation et Analyse du Discours [En ligne], 7| 2011, mis en ligne le 15 octobre 2011, consulté le 23 septembre 2019. URL : http://journals.openedition.org/aad/ 1230 ; DOI : 10.4000/aad.1230

Ce document a été généré automatiquement le 23 septembre 2019

\section{cc)}

Argumentation \& analyse du discours est mis à disposition selon les termes de la licence Creative Commons Attribution - Pas d'Utilisation Commerciale - Pas de Modification 4.0 International. 


\title{
Les esclaves dans les petites annonces des journaux brésiliens au dix-neuvième siècle : discours et idéologie
}

\author{
Slaves and Classified Ads in 19th Century Brazilian Newspapers: Discourse and \\ Ideology
}

Helena Nagamine Brandão

\section{NOTE DE L'ÉDITEUR}

Cet article a paru en portugais chez Ramos, J.M. \& M. A. Alkimin (éds). 2007. Para a História do Português Brasileiro. Estudos sobre mudança lingüística e história social, vol.5 (Belo Horizonte : Ed. FALE/UFMG)

\section{Introduction}

1 Les petites annonces, on le sait, ont un caractère documentaire : par les informations qu'elles font circuler, par leurs offres de produits et de services, elles décrivent l'univers des objets et les préoccupations d'un groupe social déterminé à une époque donnée. Dans la mesure où il fait partie de la vie quotidienne du citoyen et que sa présence s'affirme dès la mise en circulation des premiers journaux imprimés, ce genre de discours fournit un matériau qui permet d'appréhender divers aspects de la vie sociale d'une communauté discursive donnée.

2 Le présent travail analyse des petites annonces parues dans des journaux de l'État de São Paulo qui ont été collectées par des chercheurs du projet collectif Para uma História do Português Brasileiro [Pour une Histoire du portugais brésilien] et publiées sous la direction 
de Guedes et Berlinck 2000. Pour analyser ces annonces, on prendra en compte les présupposés théoriques de l'Analyse du Discours qui articule les dimensions linguistique et historique; on pourra ainsi retracer quelques aspects de l'histoire sociale du portugais de São Paulo en examinant les formes textuelles-énonciatives par lesquelles le discours se concrétise. Le corpus est constitué d'annonces collectées dans six journaux de l'État de São Paulo qui circulèrent tout au long du $19^{\mathrm{e}}$ siècle.

3 À partir du concept de genre de discours (que Bakhtine caractérise en tenant compte de trois facteurs : thème, composition, style), nous avons choisi comme premier critère d'analyse le contenu véhiculé dans les annonces. Leur relevé a montré l'hétérogénéité des objets thématisés (offre de produits, animaux, services, affaires, communiqués de caractère social et institutionnel, éducation, culture). La quantité d'annonces concernant l'esclavage a cependant attiré notre attention: elle constitue plus d'un quart du total collecté. Faisant partie du dicible, du «narrable» et de l'» argumentable» des pratiques discursives d'une communauté du $19^{e}$ siècle, la thématisation de l'esclavage dans les annonces fait de celui-ci un objet de discours. Elle offre la représentation verbale d'une façon de vivre et de penser le monde, reflétant et réfractant non seulement le quotidien, l'univers des petites informations qui circulent dans le réseau tissé par les pratiques de langage, mais aussi tout un cadre social, politique et idéologique propre à une période de l'histoire du Brésil. C'est ainsi que les annonces datant des années 1828 à 1879 englobent une période essentielle dans le combat politique mené entre le discours esclavagiste et le discours abolitionniste.

\section{Conditions historiques de production des énoncés}

4 Quelles sont les circonstances qui ont rendu possible l'émergence de ces types d'énoncés? En vue de décrire les conditions de production de ce discours, nous présenterons brièvement un panorama historique du cadre social, politique et économique en vigueur au $19^{\mathrm{e}}$ siècle.

5 Le Brésil a une longue tradition esclavagiste, car, d'après Costa (1998), «l'esclavage a marqué les destins de notre société » dans la mesure où il a déjà été mis en place dans notre pays au $16^{\mathrm{e}}$ siècle, lors des découvertes. À ce sujet, Novais (1998) note :

La mise en place de l'exploitation coloniale de l'époque moderne, de par ses déterminations politiques et économiques, renfermait en elle l'obligation du travail comme l'une de ses composantes structurelles; et l'Amérique portugaise a été, comme on le sait, l'undes espaces où cette composante a été poussée à l'extrême. configurant ainsi l'asservissement. Les populations apparaissent donc clivées en deux strates : ceux qui sont contraints au travail et ceux qui les y contraignent, les dominateurs et les dominés, les seigneurs et les esclaves (Novais 1998 : 27).

6 Ainsi, l'esclavage a déjà été instauré par le système colonial comme forme d'exploitation des terres découvertes par le colonisateur ; on a contraint d'abord les Indiens, puis les Noirs, aux travaux forcés :

Pendant plus de trois siècles, ils utilisèrent le travail des esclaves avec une intensité plus ou moins grande, dans presque tout le domaine colonial. Quand ils ne réduisaient pas les Noirs en esclavage, ils trouvaient un moyen d'obliger les Indiens à des travaux forcés. Dans les zones de mines, dans les plantations, dans les ports, l'esclave représenta, dans de nombreuses régions, la principale force de travail (Costa, $1998: 17$ ). 
7 Le $19^{\text {e }}$ siècle, stimulé par un nouvel ordre économique provoqué surtout par la Révolution industrielle et par l'apparition de nouvelles formes de capitalisme dans les pays de l'Europe occidentale, assistera à de " profondes modifications dans le système colonial », avec l'indépendance juridique des colonies américaines :

Mais la plupart d'entre elles continuèrent à être subordonnées économiquement aux pays plus développés et à être liées, en grande partie, à des solutions économiques traditionnelles. La liquidation du système colonial en Amérique est un long processus qui, sous certains aspects, existe encore de nos jours. ${ }^{1}$ La disparition du système esclavagiste est l'une des étapes de ce processus (Costa, 1998 : 18).

8 Au début du $19^{\mathrm{e}}$ siècle, sous l'influence d'idées libérales et dirigé par l'Angleterre, le mouvement anti-esclavagiste se développa et eut de fortes résonances au Brésil, ce qui finit par causer l'interdiction du trafic d'esclaves en 1831. Néanmoins, en raison de la nécessité d'une main-d'œuvre dans les plantations de café, le trafic continua sous «forme de contrebande jusqu'en 1850, où une nouvelle législation l'interrompit définitivement ». La lutte anti-esclavagiste se poursuivit avec de violentes disputes dans le domaine politico-économico-idéologique, parvenant peu à peu à des conquêtes partielles comme celle de 1871, avec la promulgation de la loi du Ventre Libre, en $1884^{2}$, la loi des Sexagénaires ${ }^{3}$ et finalement, en 1888, à l'abolition totale de l'esclavage avec la loi d'Or.

9 Après avoir présenté ce bref panorama et en nous appuyant sur ces balises historiques et culturelles, nous verrons comment le réel de l'Histoire se présente dans le réel de la langue, c'est-à-dire comment les annonces concernant l'esclavage produisent du sens et font circuler des discours sociaux, dans leur compatibilité ou dans leur incompatibilité, dans leurs relations d'alliance ou d'antagonisme, dans leurs effets d'hégémonie, de consensus ou d'hétérogénéité.

\section{Instances énonciatives}

10 Tout énoncé montre un monde dont la représentation s'effectue en fonction du rituel discursif propre au genre dans lequel il s'inscrit. En d'autres termes, le dire et le dit (le modus et le dictum des Anciens), la thématique et le régime énonciatif doivent se constituer dans des gestes solidaires qui se légitiment réciproquement. Ainsi, en tant qu'annonces véhiculées dans des journaux, ces énoncés obéissent à des contraintes semblables à celles dont on faisait usage à l'époque. Les annonces avaient pour objectif d'informer les lecteurs d'un événement donné (fuite/offre/location d'esclaves) et visaient un effet perlocutoire (capture/achat). Mais celui qui parle dans le domaine de l'espace social a-t-il le droit de parler de la manière dont il énonce ce qu'il dit ? Pensons à Foucault (1969) lorsqu'il se réfère au discours médical :

Qui parle? Qui, dans l'ensemble de tous les individus parlants, a l'autorité d'exercer cette espèce de langage ? [...] Le discours médical ne peut venir de n'importe qui, sa valeur, son efficacité, ses propres pouvoirs thérapeutiques et, d'une manière générale, son existence comme discours médical ne sont pas dissociables du personnage statutairement défini qui a le droit de l'articuler (65).

11 Et qui est l'autre à qui je parle de cette manière ? Dans quelle position discursive le JeTude la deixis discursive se place-t-il ? Ces annonces sont rédigées, presque dans leur totalité, sur le plan énonciatif du récit (Benveniste 1966), avec une structure semblable à celle de la nouvelle: le Je du locuteur s'efface et met l'accent sur l'événement. Cependant, le locuteur est celui qui a fait passer l'annonce et il apparaît comme : 
Reverendissimo Doutor [Révérend Docteur] Joaquim Manoel Gonçalves de Andrade, Capitão [Capitaine] José Pereira de Queiroz, Alferes [Sous-lieutenant] X, Sargento Mór [Sergent chef] Y, etc. Comme on peut le remarquer, on ne mentionne pas seulement le nom complet, mais aussi les titres ou les titres honorifiques qui les distinguent socialement : ce sont les propriétaires des esclaves, ceux qui sont du côté du pouvoir institué, du pouvoir dominant. Par conséquent, leurs discours sont autorisés et légitimes. Ils n'émergent dans le réseau du discours social en vigueur que parce qu'ils émanent du pouvoir institué par la société esclavagiste. C'est ce pouvoir qui confère à ces acteurs sociaux la capacité à capturer l'esclave qui s'est enfui de chez lui, de dénoncer et ramener le fugitif qui a été retrouvé, de faire appel aux autorités policières. Par exemple :

(1) De chez le Tenente [Lieutenant] Luiz Francisco da Costa, demeurant à Rio de Janeiro, rua São Francisco de Paula numéro 90, a disparu sans aucune raison le 1 er juillet de l'année en cours, un de ses esclaves rusés du nom de Francisco, de la nation Benguella, âgé de 19 à 20 ans, avec une barbichette, édenté à la partie supérieure ; mais avec une seule dent de devant à la même partie, nez plat, lèvres charnues, costaud, pieds et jambes bien formés, tout maigre, les pommettes saillantes, au coin du front une petite bosse comme une petite graine de haricot qui s'est déplacée: on prie toute personne qui donnera de ses nouvelles, ou qui l'emmènera chez son seigneur, ou à la ville de São Paulo chez Manoel Gonçalves Pereira, au village de Sanctos chez João da Monte Bastos, au village d'Ytu chez le prêtre Felis do Amaral Grugel et au village de Sorocaba chez Bento José Ribeiro, non seulement on lui paiera les dépenses, mais on lui offrira aussi une gratification pour son travail (O Farol Paulistano, 16 février 1828).

(2) Le $1^{\text {er }}$ jour du mois courant, s'est enfui de chez le Capitão [Capitaine] José Pereira de Queiroz du village de Jundiahy un esclave créole du nom de João Pedro, charpentier, taille moyenne, corps chétif, barbe fournie, et il a eu un coup sur deux doigts de la main gauche, dont les ongles sont déformés, dont les doigts sont près du grand doigt. $25 \$ 600$ réis ${ }^{4}$ sont offerts en récompense à celui qui le conduira jusqu'à cette ville chez le Capitãojosé Rodrigues Pereira ou jusqu'au village de Jundiahy chez son seigneur (O Farol Paulistano, 13 décembre 1828)

(3) Au début du mois de février de cette année 1841, deux esclaves se sont enfuis de la fazenda de Sua Excelência Reverendíssima [Son Excellence Révérend]. Un dénommé Eleutherio, créole, marié, et qui a été l'esclave de Francisco Rodrigues, demeurant à Bom-Successo, Freguezia de Nossa Senhora da Conceição dos Guarulhos : signes particuliers : grand, maigre, visage allongé, une barbiche, long cou, un goitre pas très grand, les dents de devant pourries, des jambes longues et fines, de grands pieds et un doigt cassé à l'une des mains, c'est un Noir qui travaille dans les champs, mais très bon terrassier, âgé de 40 ans environ, un peu plus ou un peu moins. Joaquim, nation-Congo, taille moyenne, visage tout grêlé par la variole [en portugais « bexigoso »], c'est pourquoi on l'appelle Joaquim Bexiga, grosses jambes et gros pieds, mais qui ne sont pas enflés, il n'a pas de barbe et il doit avoir trente ans, un peu plus ou un peu moins, et il a travaillé autrefois dans le Jardim Botanico de cette ville. Ils portent tous deux un pantalon et une chemise en coton épais. À toute personne qui les capture, en plus des dépenses contractées avec la prison, on promet une gratification de $12 \$$ réis pour chacun d'eux et nous jurons également que nous intenterons un procès, conformément à la loi, à celui qui les fouettera ou les gardera en son pouvoir. Dans cette ville, ils pourront les remettreau Reverendíssimo Doutor Joaquim Manoel Gonçalves de Andrade, et à la fazenda à celui qui la dirige (À Phenix, 13 mars 1841).

(4) On gratifie : S'est enfui de la fazenda - Morro Azul le mulâtre à la peau claire, du nom de Paulo, appartenant à Silverio Rodrigues Jordão, ayant les signes particuliers suivants : cheveux raides, oreilles décollées, mauvaises dents, poil follet, peu de barbe au menton, cicatrice de blessure au cou, une autre de hache à l'un des pieds, 
de petite taille et aux pieds plats, on soupçonne qu'il se trouve dans cette ville. Celui qui le capturera et qui le ramènera au soussigné sera bien récompensé. Luiz Pinto Homem de Menezes (Correio Paulistano, 21 février 1879).

(5) Les esclaves ci-après ont été recueillies, dans la Prison du Village de Sanctos, capturées dans le Quilombo ${ }^{6}$ récemment vaincu, dont les noms et les seigneurs sont déclarés conformément à la confession des esclaves. Maria, de la nation Benguela, esclave de Bernardo Guedes; Eva, créole, esclave du Alferes [Sous-lieutenant] Antonio Galvão; Anna, créole, esclave de Francisco Pereira Mendes; Domingas, Angola, esclave du Capitão Joaquim (surnommé Inhoquim) (O Farol Paulistano, 7 juin 1828).

(6) Il existe dans le village de Parahibuna, dans cette province, un esclave qui affirme appartenir au Senhor Doutor [Seigneur Docteur] Penteado, demeurant à Casa-Branca ou Mogy-mirim. Si ce Seigneur Docteur a effectivement un esclave qui s'est enfui, il peut s'adresser au Comendador [Commandant] Marcellino José de Carvalho dans ce village-là (O Constitucional, 23 juin 1854).

(7) Un appel à la police. Se sont enfuis de chez le soussigné, de sa fazenda des Piteiras à Mogy-mirim, dans la nuit du 18 au 19 du mois courant, deux esclaves dont les noms et les signes particuliers sont les suivants: Miguel, noir de 30 à 40 ans, taille et corps moyens, barbu, créole du Rio Grande do Sul, parle avec un accent africain et se dit maçon. Segismundo, métis, 20 ans, un peu plus ou un peu moins, petite taille, corps gros et vigoureux, pieds et mains plats aux orteils et aux doigts courts, petite moustache, porte des vêtements fins et a emporté une montre en argent. À ceux qui les arrêteront, on offrira une gratification de $50 \$ 000$ réis à chacun d'eux; nous jurons que nous intenterons un procès à ceux qui les hébergeront. Mogy-mirim, le 29 mai 1879 Antonio Joaquim de Freitas Leitão (Correio Paulistano, $1^{\mathrm{er}}$ juin 1879).

Le statut du discours dominateur ne peut avoir comme interlocuteur que quelqu'un qui appartienne au même cercle social, politique, économique; c'est un discours hégémonique, qui exclut et ne dialogue qu'avec ses pairs. L'allocutaire représente les autres propriétaires d'esclaves (ou leurs représentants, tels que l'intendant, le capitão do $\operatorname{mato}^{7}$ ), ceux qui ont accès au journal (aux biens culturels), les autorités instituées.

13 L'esclave est le référent, l'objet de la thématisation de ce discours. C'est le Il, la nonpersonne à laquelle se réfère Benveniste. Exclu de la relation de personne, il est en dehors de toute interlocution possible et, par conséquent, de toute relation d'intersubjectivité. Dans ce régime énonciatif de domination, l'esclave est devenu objet : en reflétant et en réfractant la réalité, le langage, dénonce sa condition de nonsujet.

[...] la continuité de l'esclavage lors de la jeune monarchie s'est fondée sur le droit positif: le droit de propriété des seigneurs sur leurs esclaves, ceux-ci étant assimilés juridiquement à de simples marchandises. Donc, du point de vue légal, on vidait la relation esclavagiste de son lien seigneurial pour mettre en évidence son sens commercial. Ce faisant la monarchie exacerbait - en principe - le pouvoir privé des seigneurs sur leurs prisonniers, transformé en simple droit de propriété (Castro, $1997: 341$ ).

14 L'annonce ci-dessous, sous forme de lettre, illustre bien la condition de chose à laquelle étaient réduits les esclaves :

(8) Hier matin, on m'a envoyé un Noir de la Guinée, très grossier et habillé à la manière de ceux qui viennent en groupe et on m'a dit qu'on l'a attrapé alors qu'il errait dans la rue comme s'il était perdu. Avec l'aide d'uninterprète, j'ai appris seulement qu'il n'était pas encore baptisé et qu'il s'était égaré lorsqu'il était sorti pour couper du bois : veuillez donc Votre Grâce insérer cette annonce dans votre feuille pour que son maître apparaisse, sur quoi je déclare que s'il ne se présente pas dans les 15 jours à compter de la publication de la feuille, je dois le remettre à la 
Provedoria dos Residuos [Division des Résidus] ; à laquelle il appartient de reconnaître les choses dont on ne connaît pas le propriétaire. - São Paulo, le 9 avril 1830 - Le Juge de Paix Suppléant de la Freguezia da Sè. José da Silva Merceanna ( $O$ Farol Paulistano, 24 avril 1830).

Comme objet d'usage et déterminé par l'»obligation du travail» (celle dont parle Novais), l'esclave devient un produit, un bien nécessaire de la société esclavagiste, un indice de statut social et économique des propriétaires. C'est dans cette condition d'objet, de marchandise dans laquelle on avait investi un capital dont on espérait le retour sous forme de profit que les esclaves devaient être capturés quand ils s'enfuyaient ou qu'ils pouvaient être vendus, loués, comme l'attestent les énoncés cidessous :

(9) On vend une Noire rusée qui sait faire la cuisine, les tâches habituelles d'une maison, fait la lessive, repasse très bien et fait la couture de manière très passable. Que celui qui a l'intention de l'acquérir se rende à la rua da Quitanda au magasin de Domingos José Vieira, pour la voir et conclure l'affaire, et encore six Noirs de la nation Banguela, et dans le même magasin il $\mathrm{y}$ a une quantité de très bons raisins secs arrivés récemment et à de très bons prix ( $O$ Farol Paulistano, 20 septembre 1828).

(10) On loue une femme métisse pour tout service de maison, sachant bien repasser et faire la cuisine. Conclure l'affaire rua do Ypiranga numéro 2 (Correio Paulistano, 12 février 1879).

Inscrit dans le langage comme Il, donc référent, hors du circuit de l'interlocution Je-Tu, la langue dénonce le statut de l'esclave comme non-personne: en opposition au locuteur qui est identifié par son nom complet généralement suivi du titre, indiquant sa condition de citoyen et son statut social, l'esclave, quand on le nomme, n'est désigné que par ce qu'on coutume d'appeler prénom: «s'est enfui un esclave du nom d'Alexandre, Francisco, Maria, Eva, Domingas, Benedito, etc. ». Le nom est le premier pas d'un processus symbolique de construction de l'identité: le nom distingue, singularise, individualise, il confère un statut d'existence à l'être désigné. Mais pour l'esclave, objet réduit à la condition de simple marchandise, un prénom suffisait.

Néanmoins, cet immense contingent anonyme de Marias, Joaquins, Beneditos, Franciscos, etc. devaient être identifiés quand ils s'enfuyaient. Comment? Par la description des signes particuliers de leur corps, par les vêtements qu'ils portaient; c'est-à-dire qu'à l'identité en tant que signe qui individualise et qui confère la citoyenneté se substitue le signalement, selon les termes de Bakhtine. D'après ce dernier, le signe est vivant, dialectique, dynamique et pour être compris, il exige une attitude responsive active, tandis que le signal est inerte et ne sert que pour être reconnu. Si nous transférons ces concepts à notre cas, l'esclave, en tant qu'objet, n'est pas perçu par l'agent de ce discours dominateur comme le partenaire d'une interlocution, mais comme marchandise, élément de transaction commerciale, investissement pour l'obtention du profit, et ce qui le distingue des autres, ce sont les marques du corps. D'où la présence des descriptions, généralement fort détaillées, de ces signes particuliers. En plus des annonces déjà citées, examinons celles-ci :

(11) De chez le Coronel [Colonel] Ignacio de Sá du village de Coriba, s'est enfui un esclave noir du nom de João, de bonne apparence, stature, grands yeux, et handicapé de la main gauche à cause de deux coups qu'il a reçus à la jointure de la dite main, qui a failli être amputée des trois doigts qu'il ne peut pas contrôler [...] ( $O$ Farol Paulistano, 8 novembre 1828).

(12) S'est enfui le 2 septembre 1829 un Noir du nom d'Antonio, de la nation Congo, taille moyenne, trapu, a l'air d'avoir 20 ans, barbe peu fournie, pieds fins, sur la 
poitrine, du côté droit ou gauche, est marquée la lettre B; il portait une chemise rayée foncée et un pantalon en tissu bleu [...] (O Farol Paulistano, 24 octobre 1829).

(13) $100 \$ 000$ de gratification à celui qui capturera, donnera des nouvelles exactes ou ramènera dans cette ville [...] au soussigné, un gamin du nom de Jacinto, âgé de 19 à 20 ans, bien noir, avec une petite moustache, costaud et au corps bien fait, sauf les pieds qui sont mal faits, aux gros orteils tournés vers l'intérieur, aux ongles abimés par des bestioles qu'il a eus quand il était petit, et l'un des orteils porte la marque d'une ancienne plaie qu'il a eue il y a longtemps, il est très malin et intelligent, parle très rapidement et bégaie et quelquefois, il a du mal à parler et c'est pour cela qu'il parle de manière impulsive et très rapidement, il a le nez bien aplati, les yeux rougeâtres [...] il s'est enfui le 13 décembre 1851 [...] (O Constitucional, 4 juin 1853).

(14) [...] se sont enfuis deux esclaves du nom de José dans la nuit du 16 au 17 février de l'année 1853 en cours ; et leurs signes particuliers sont: l'unde la nation Mozambique, âgé de 46 ans environ, taille moyenne, trapu, visage rond, dents pointues, barbu, chauve et avec une grosse bosse sur la tête chauve (ce qui est très caractéristique), rusé et actif. Il a des marques de châtiment sur le dos et il en a aussi sur les fesses. L'autremulâtre, âgé de 20 ans environ, taille moyenne, trapu, visage rond, petit front, petites oreilles et petite bouche, sans barbe, noir et luisant, a l'air très bête au premier abord parce qu'il parle lentement, mais il ne l'est pas, il est rusé. Il a les pieds, et surtout l'un d'eux, quelque peu enflés et les orteils sont presque sans ongles, il semble que cela vient de bubons. Il ne marche pas avec désinvolture, mais plutôt en chancelant, ne court presque pas ( $O$ Constitucional, 2 juillet 1853).

(15) Esclave fugitif $400 \$ 000$ de gratification. De chez le soussigné s'est enfui son esclave, du nom de Vicente, métis, âgé de 32 ans, cheveux frisés, mais pas crépus : bonne allure, poitrine et épaules larges, mais ces dernières un peu hautes; se tient droit quand il marche, mais il le fait avec difficulté car il a les pieds plats qui tournent, comme ceux qui ont des durillons, monte bien à cheval, peut se présenter comme bouvier, car il sait prendre les chevaux au lasso ou comme cocher; il a emporté de bons vêtements pour être bien habillé, parle assez bien et avec un accent provincial, lit et écrit un peu et il a un dentier, car il lui manque les dents du haut. Il peut se présenter comme libre et on peut facilement le croire (Correio Paulistano, 22 juillet 1879).

(16) De chez Dom Jeronymo Rabassa s'est enfuie une esclave cabra ${ }^{8}$, du nom d'Iria, âgée de trente-quatre ans plus ou moins, déjà sans dents en haut et en bas quelques dents gâtées, elle a le pied droit plus enflé que le pied gauche et une cicatrice au front. On prie celui qui sait où elle se trouve de se rendre à la rua do Rosario, à la maison numéro 29 , si la nouvelle est véridique, cette personne sera largement récompensée (O Farol Paulistano, 11 avril 1829).

18 Que «disent» ces descriptions? La population esclave la plus fréquente qui apparaissait dans ces annonces était composée : a) d'hommes qui constituaient la plus grande partie $d u$ contingent d'esclaves justement à cause du travail lourd qu'ils devraient exécuter ; d'après Costa (1997), la proportion entre les esclaves hommes et les esclaves femmes était de quatre à une ; b) d'adultes entre 20 et 35 ans parce que c'était la force de travail la plus productive.

La description des signes particuliers indique encore une population constamment en proie à des problèmes de santé, précocement vieillie, avec des handicaps physiques qui semblent provenir de châtiments corporels.

Dans le régime d'esclavage, où le travail se démoralise et résulte d'une imposition, le groupe dominant se voit fréquemment obligé de recourir à la violence physique quand il veut accomplir ses desseins. Pour maintenir le rythme de travail, pour empêcher des attitudes d'indiscipline ou pour réprimer des révoltes, pour terroriser les esclaves, les maintenir humbles et soumis, pour éviter ou punir des évasions, les seigneurs avaient recours aux types de châtiment les plus divers, car 
les accords et les réprimandes ne valaient pas grand-chose. On ne concevait pas d'autre manière de réguler la prestation de services et la discipline de l'esclave. Ce que l'on pouvait condamner, c'était l'excès, l'abus commis par quelques seigneurs ou leurs mandataires, les intendants'. Le châtiment physique s'imposait, selon l'opinion de l'époque, comme la seule mesure coercitive efficace. La conviction que beaucoup d'esclaves ne travaillaient que s'ils étaient dûment roués de coups s'était généralisée (Costa, 1997 : 337).

L'annonce (12), par exemple, montre une pratique habituelle chez les propriétaires : celle de marquer le corps de l'esclave au fer rouge avec ses initiales.

\section{Composition textuelle/discursive des annonces}

\subsection{Caractéristiques formelles}

On observe une certaine homogénéité par rapport aux formes de textualisation de ces annonces dont la structure peut être schématisée de la façon suivante :

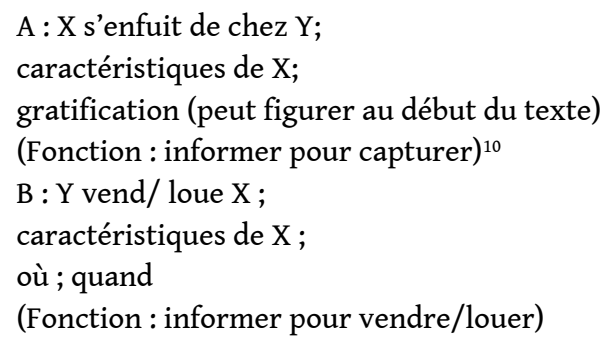

Les annonces du premier type (a) ont une caractéristique informative : elles focalisent l'attention sur le Il - l'esclave, l'objet thématisé ; tandis que celles du deuxième type (b) ajoutent à la fonction informative des indices de la fonction persuasive : en focalisant l'attention sur le $\mathrm{Tu}$ - l'acheteur; elles cherchent à promouvoir, à qualifier positivement le produit (l'esclave) à vendre ou à louer. Contrairement à celles-ci, les annonces du type (a) cherchent à présenter l'objet, en le décrivant dans son apparence réelle, avec les traits les plus objectifs possibles, nécessaires à son identification immédiate. Les annonces du type (b) contiennent déjà embryonnairement quelques caractéristiques que les annonces adoptent ultérieurement jusqu'à la sophistication maximale d'aujourd'hui: glissement de la fonction référentielle à la fonction persuasive (du Il vers le Tu), adoptant des stratégies d'argumentation et de séduction.

(17) ESCLAVES. Qui a l'intention d'acheter : 1 métis, âgé de 16 ans, présentant les meilleures conditions pour s'occuper d'enfants. 1 créole, 22 ans, cuisinier, sachant laver et repasser et qui parle pratiquement très bien l'allemand. 1 ayant soi-disant 10 ans ; tous en bonne santé et beaux. Que celui qui a l'intention de les acquérir se rende rua da Imperatriz, au Grand Hôtel de l'Europe, pour voir et conclure l'affaire avec son maitre, qui doit prendre le vapeur qui le 30 du mois courant doit partir de Santos vers le sud, mais cela ne l'empêchera pas de conclure une bonne affaire avec l'acheteur (Correio Paulistano, 23 janvier 1879).

(18) ESCLAVE. On vend une belle femme de chambre, sans aucun défaut, âgée de 16 ans, la raison de sa vente va plaire à l'acheteur. Rua do Seminario dos Educandos numéro 4 (Correio Paulistano, 24 juin 1879).

(19) BONNE AFFAIRE! On vend un esclave bon pour tous travaux des champs, robuste et en bonne santé, à voir et à traiter l'affaire rua da Imperatriz numéro 52 (Correio Paulistano, 20 septembre 1879). 
(20) ON VEND 14 esclaves des deux sexes, il y a un beau créole majordome et une créole de 15 ans qui lave, repasse et fait la cuisine. Affaire à traiter rua da Constituição numéro 14 (Correio Paulistano, 22 novembre 1879).

(21) ESCLAVES DANS LA TRAVESSA Paisandù numéro 1. Il y a 25 esclaves en bonne santé et robustes à vendre à des prix raisonnables; que celui qui a l'intention d'en acheter se rende jusqu'à cette maison-là (À Constituinte, 17 octobre 1879).

\subsection{Hétérogénéité discursive : sous l'« un », l'« autre »} du langage, sa constitution hétérogène. Pour l'Analyse du Discours, d'après le principe de dialogisme proposé par Bakhtine, chaque mot est pluri-accentué, il est traversé par l'autre ; c'est-à-dire qu'une formation discursive n'est pas un bloc homogène, stable, mais un domaine ouvert et inconscient. Cette orientation dialogique n'est pas limitée aux énoncés qui portent la marque de la citation, de l'allusion, etc., ni à un autre réductible à une image d'interlocuteur.

Dans l'espace discursif, l'Autre n'est ni un fragment localisable, ni une citation, ni une entité extérieure. Il n'est pas nécessaire qu'il soit repérable par quelque rupture visible de la compacité du discours. Il se trouve à la racine d'un Même toujours déjà décentré par rapport à lui-même, qui n'est à aucun moment envisageable sous la figure d'une plénitude autonome. Il est ce qui fait systématiquement défaut à un discours et lui permet de se fermer en un tout. Il est cette part du sens qu'il a fallu que le discours sacrifie pour constituer son identité (Maingueneau, $1984:$ 31).

Donc, la relation avec l'autre doit être perçue indépendamment de n'importe quelle forme d'altérité marquée. On l'étend plus encore en concevant cet autre non pas comme une présence qui se manifeste soit explicitement soit implicitement, mais comme une absence, comme un manque, comme l'interdit du discours. À savoir, toute formation discursive dans l'univers du grammaticalement dicible circonscrit la zone $\mathrm{du}$ dicible légitime, définissant l'ensemble d'énoncés qui peuvent être mis à jour dans une énonciation donnée à partir d'un endroit déterminé. Ce faisant, elle circonscrit aussi une zone de non-dicible, définissant l'ensemble des énoncés qui doivent être absents de son espace discursif ; elle délimite de cette manière le territoire de l'autre qui apparaît comme incompatible, en l'excluant de son dire.

Les énoncés présentent, de cette façon, une double face: un "endroit» et un "envers", qui sont indissociables. Il incombe à l'analyste de les déchiffrer non seulement dans leur "endroit ", en les mettant en rapport à leur propre formation discursive, mais aussi dans leur " envers ", en examinant la face cachée où se dissimule le rejet du discours de l'autre. Ce qui revient à dire qu'il incombe à l'analyste d'appréhender non seulement une formation discursive, mais aussi l'interaction entre des formations discursives, vu que l'identité discursive se construit dans la relation avec un autre présent linguistiquement ou non dans l'intradiscours.

Revenant aux textes analysés, qu'est-ce qui se cache sous l'apparente homogénéité de la superficie linguistique? Quel est l'autre, le non-dit qui s'insinue dans la formation discursive esclavagiste dont ces annonces sont la matérialisation? L'interdit porte sur la parole de l'esclave qui, ne pouvant se manifester discursivement, fait du silence son arme, ou de l'évasion l'une des manières de résister à la domination.

Insurrections, crimes, fuites, suicides, travaux accomplis mal ou avec lenteur,

l'obstination à résister à des ordres donnés, c'étaient là les moyens dont disposait 
l'esclave pour se manifester contre la situation dans laquelle il était maintenu [...]. De tous les mécanismes de protestation, le plus fréquent fut l'évasion. [...] Quand on le rattrapait, le fugitif était reconduit à son maître et sévèrement puni pour que cela puisse servir d'exemple aux autres. Attaché au tronc de l'arbre et fouetté, il portait ensuite de lourds anneaux aux pieds et aux mains ou plus rarement, un carcan. Rien n'était suffisant pour arrêter les évasions. Il y avait des esclaves qui s'enfuyaient deux, trois fois et qui répétaient cette prouesse dès que possible (Costa, 1997 : 367-368).

Dans le sémantisme du mot fuir - « s'éloigner ou se retirer hâtivement pour échapper à quelqu'un ou à un danger, se retirer à la hâte, se débarrasser de " (d'après le dictionnaire Aurélio) -, se trouve le sens de l'action des esclaves : en même temps qu'ils s'enfuient pour échapper au danger, ils manifestent aussi par cet acte une forme de résistance ; résistance primitive, il est vrai, instinctive, animale, mais la seule possible pour échapper au système oppresseur. L'impression que l'on a, c'est celle d'un quotidien conflictuel où les propriétaires et les esclaves sont constamment en état d'alerte : les uns cherchant à maintenir le système au moyen de la répression et de la violence et les autres, les plus faibles, cherchant, sous la forme de micro-résistances (Michel de Certeau), à vider, à miner ce système oppresseur.

Plus l'agitation sociale augmentait, plus la confusion qui régnait s'accroissait - les chocs entre les seigneurs et les esclaves, l'agitation dans les senzalas ${ }^{11}$, les évasions d'esclaves - plus on forçait le changement de la situation et on préparait le climat pour la mesure décisive qui aurait pour résultat la suppression définitive de l'esclavage (Costa 1997 : 347).

Ainsi, à leur manière, et avec les armes dont ils disposaient, les esclaves créaient des formes de résistance au système d'oppression, contribuaient à le miner et l'obligeaient à procéder à des changements ${ }^{12}$. Parmi les annonces collectées quelques-unes portaient déjà la marque des changements qu'une inévitable abolition de l'esclavage allait opérer dans l'univers du travail et, en conséquence, dans le panorama social : l'embauche de la main d'oeuvre libre sous forme d'immigration (européenne et asiatique) :

TRAVAILLEURS! Viennent d'arriver d'Allemagne et souhaitent se faire embaucher ici ou ailleurs, 2 Boulangers, 2 Garçons de comptoir, 1 Scieur, 7 Mineurs, lesquels ne doutent pas qu'ils vont accepter n'importe quel autre travail qu'on leur offrira. Que celui qui veut utiliser leur service se rende au Consulat, Rua de São Bento numéro53 (Correio Paulistano, 27 juin 1879).

COLONS PORTUGAIS, Rua da Boa Vista 47A - Escriptorio Menezes \& Companhia [Bureau Menezes \& Compagnie]. Font venir des colons portugais pour le labourage garantissant le plus grand soin pour le choix et se chargeant du contrat. Les intéressés trouveront tous les renseignements dont ils auront besoin dans notre bureau (Diário Popular, 12 novembre 1884).

\section{BIBLIOGRAPHIE}

Angenot, Marc. 1984. «Le discours social : problématique d'ensemble », Bourque, Gilles et al. (éds). Le discours social et ses usages. Cahiers de recherche sociologique 2. 1, 19-44 
Bakhtine, Mikhaïl. 1984 [1952-1953]. « Les genres du discours - Problématique et définition », Esthétique de la création verbale (Paris : Gallimard), 277-326

Brandão, Helena N. 1991. Introdução à Análise do Discurso (Campinas, SP : Ed.

UNICAMP)

Castro, Hebe M. M. de. 1997. « Laços de família e direitos no final da escravidão », Alencastro, Luiz F. de (éd). História da vida privada no Brasil. Vol. 2 (São Paulo : Cia das Letras), 337-383

Certeau, Michel de. 1990. L'invention du quotidien. 1. Arts de faire, éd. Luce Giard (Paris : Gallimard)

Costa, Emília V. da. 1998. Da senzala à colônia. $4^{\mathrm{e}}$ éd. (São Paulo : Fundação Editora UNESP)

Foucault, Michel. 1969. L'archéologie du savoir (Paris : Gallimard)

Guedes, Marymarcia \& Rosane de A. Berlinck (éds). 2000. «E os preços eram commodos... Anúncios de jornais brasileiros - Século XIX » (São Paulo : FFLCH/USP: Humanitas)

Maingueneau, Dominique. 1989. Nouvelles tendances en analyse du discours (Paris : Hachette)

Maingueneau, Dominique. 1984. Genèses du discours. (Bruxelles : Mardaga)

Novais, Fernando A. 1998[1997]. « Condições da privacidade na colônia », Mello e Souza, Laura de (éd.). História da vida privada no Brasil : cotidiano e vida privada na América portuguesa 1 (São Paulo : Cia das Letras), 13-39

\section{NOTES}

1. Pour corroborer cette affirmation de l'auteure, on trouve encore de nos jours dans les journaux des dénonciations de la pratique de l'esclavage parmi nous, héritage de la culture coloniale, malgré le travail du gouvernement fédéral, comme cette nouvelle envoyée par son Service de Communication : «Le gouvernement combat le travail esclave. L'abolition de l'esclavage au Brésil eut lieu en 1888, mais d'après la Comissão Pastoral da Terra [Comission Pastorale de la Terre] (CPT), 25.000 personnes sont soumises à des conditions analogues au travail esclave au Brésil » (http://www.brasil.gov.br/emquestao, consulté le 24/09/2003).

2. La loi dite de Rio Branco ou du «Ventre libre» stipulait que tous les enfants naîtraient désormais libres et resteraient seulement jusqu'à leur majorité au service du maître de la mère pour l'indemniser des frais d'éducation. Le recrutement de l'esclavage était ainsi tari dans l'une de ses sources, la naissance (NdR).

3. La Loi Saraiva-Cotegipe, ou Loi des Sexagénaires est née d'un projet de Rui Barbosa, député originaire de Bahia : elle libérait tous les esclaves de plus de 60 ans (NdR).

4. Réisest le pluriel de real, unité monétaire au Portugal, au Brésil et dans d'autres pays lusophones, pendant certaines périodes de l'histoire.

5. Fazenda: grande propriété de culture et d'élevage. Fazendeiro : grand propriétaire terrien

6. Le quilombo désigne un village ou une communauté d'esclaves en fuite dans les régions reculées du Brésil.

7. Capitão do mato : Il s'agit d'un employé chargé de poursuivre et de capturer les esclaves fugitifs.

8. Cabra : métis de Mulâtre et de Noir.

9. En portugais : feitores ou capatazes.

10. Costa (1997 : 371) indique une autre fonction des dénonciations : «Parfois, ils se disaient au service des patrons et continuaient à faire des demandes et à tirer d'autres avantages tout en utilisant le nom du seigneur. D'où le soin que prenait les propriétaires, au moment de dénoncer 
la fuite, de déclarer simultanément qu'ils déclinaient toute responsabilité des actes que les esclaves viendraient à commettre en leur nom ».

11. Senzala: Grand logement destiné aux esclaves.

12. Sur la polémique travail des esclaves $x$ travail libre, Costa $(1997: 37)$ dit : « la plupart [des fazendeiros] continuaient à croire, jusqu'à la décennie de 1880, qu'il était difficile, voire impossible, de substituer l'esclave (ceci en dépit du nombre croissant de ceux qui argumentaient en faveur de l'immigration et du travail libre). Si l'on ajoute à cela le fait que, pour la plupart des fazendeiros, les esclaves représentaient du capital déjà investi, qu'ils ne voudraient pas voir disparaître du jour au lendemain, il sera possible de comprendre pourquoi même ceux qui étaient convaincus de la supériorité du travail libre, continuaient à s'opposer à l'abolition de l'esclavage ou ne l'acceptaient qu'à condition que les fazendeiros soient indemnisés pour la perte de leur propriété ».

\section{RÉSUMÉS}

Cet article analyse un ensemble de petites annonces publiées dans des journaux brésiliens entre 1828 et 1880 . Prenant comme support théorique l'Analyse du Discours, il examine des annonces portant sur des esclaves en vérifiant comment sont représentées dans le discours les relations quotidiennes, et plus particulièrement les relations instituées par le travail des esclaves dans le régime colonial, ainsi que leurs implications sociales, politiques et idéologiques.

This paper analyzes a sample of classified ads published in Brazilian newspapers between 1828 and 1880 , and related to the slavery regime implemented in Brazil since the early colonization. Within the framework of Discourse Analysis, it examines how everyday relationships established by the compulsion of slave labor are represented in discourse with all their social, political and ideological implications.

\section{INDEX}

Mots-clés : analyse du discours, annonce, énonciation, esclavage

Keywords : classified ads, discourse analysis, enunciation, slavery

\section{AUTEUR}

\section{HELENA NAGAMINE BRANDÃO}

Universidade de São Paulo 